\title{
Acute Leukemia Presenting with Musculoskeletal Manifestations: A Case Series
}

\begin{abstract}
Introduction: Leukemia is the most common childhood malignancy accounting for $30 \%-40 \%$ of cases. Acute lymphoblastic leukemia is the most common leukemia in children with peak incidence in 2-6 years of age. The present study aims to assess the incidence of acute leukemia in patients presenting with musculoskeletal manifestations. Materials and Methods: This is a retrospective study conducted in a tertiary center from January 2014 to December 2018. A total of 63 children presented with musculoskeletal manifestations and underwent bone marrow examination. Based on final marrow diagnosis, the study group was divided into leukemic and nonleukemic groups. Results: Fever was the most common presenting complaint and was present in all the patients of both the groups. The occurrence of hepatosplenomegaly was comparatively higher in the leukemic group than in patients with juvenile idiopathic arthritis (JIA). The predominant type of arthritis was oligoarticular (68.15\%) in the leukemic group and polyarticular $(77.27 \%)$ in the nonleukemic group. Rheumatoid rash was noted in $20 \%$ of JIA patients, and none of the patients in the leukemic group had rash. The percentages of anemia, leukopenia, and thrombocytopenia were statistically higher in leukemia patients than in JA patients. Conclusion: Bone marrow studies are a prerequisite in diagnosing leukemias. However, based on the presence of few atypical clinical and laboratory features, leukemia can be excluded in JIA patients.
\end{abstract}

Keywords: Atypical presentation, childhood leukemia, juvenile rheumatoid arthritis

\section{Introduction}

Leukemia is the most common childhood malignancy accounting for $30 \%-40 \%$ of cases. $^{[1]}$ Acute lymphoblastic leukemia (ALL) is the most common leukemia in children with peak incidence in 2-6 years of age. ${ }^{[2]}$ Leukemias characteristically present with fever, bleeding manifestations, organomegaly, and deranged hematological profile. Sometimes, leukemia can show heterogeneous clinical course and present with musculoskeletal manifestations in the form of arthralgia or overt arthritis with near-normal hematological profile. Diagnosis of acute leukemia in such cases may be delayed as these may be initially misdiagnosed as juvenile idiopathic arthritis (JIA). With this background, the present study was carried out to assess the incidence of acute leukemia in patients presenting with musculoskeletal manifestations and to assess the clinical and laboratory findings that help in differentiating these two groups.

This is an open access journal, and articles are distributed under the terms of the Creative Commons Attribution-NonCommercial-ShareAlike 4.0 License, which allows others to remix, tweak, and build upon the work non-commercially, as long as appropriate credit is given and the new creations are licensed under the identical terms.

For reprints contact: WKHLRPMedknow_reprints@wolterskluwer.com

\section{Materials and Methods}

The present study was a retrospective study conducted at a tertiary center from January 2014 to December 2018. A total of 63 children presented with musculoskeletal manifestations and underwent bone marrow examination. We enrolled children below the age of 16 years in this study. Prior informed consent was obtained from the parents/guardian to perform bone marrow aspiration and biopsy studies as well as to use the information obtained for future research purposes. Based on final marrow diagnosis, the study group was divided into leukemic and nonleukemic groups. Clinical records of both the groups were reviewed, and the following data were extracted and statistically compared.

\section{Clinical data}

Age, sex, fever (axillary temperature $\geq 37.8^{\circ} \mathrm{C}$ ), lymph node enlargement, hepatomegaly, splenomegaly, type of arthritis (arthritis involving $\leq 4$ joints is oligoarthritis and $\geq 5$ is polyarthritis), and rheumatoid rash.

How to cite this article: Kesarapu S,
Vangala N, Uppin SG, Uppin MS, Paul TR,
Rajasekhar L. Acute leukemia presenting with
musculoskeletal manifestations: A case series. Indian
J Med Paediatr Oncol 2020;41:29-33.

\section{Swetha Kesarapu', Navatha Vangala ${ }^{1}$, Shantveer G Uppin ${ }^{1}$, Megha S Uppin ${ }^{1}$, Tara Roshni Paul', Liza Rajasekhar ${ }^{2}$}

${ }^{1}$ Department of Pathology, Nizams Institute of Medical Sciences, Hyderabad, Telangana, India, ${ }^{2}$ Department of Rheumatology, Nizams Institute of Medical Sciences, Hyderabad, Telangana, India

Submitted: 07-May-2019

Revised: 20-Jun-2019

Accepted: 12-Jul-2019

Published: 24-Apr-2020

Address for correspondence:

Dr. Shantveer G Uppin,

Department of Pathology,

Nizams Institute of Medical

Sciences, Hyderabad,

Telangana, India.

E-mail:drsguppin@yahoo.co.in

Access this article online

Website: www.ijmpo.org

DOI: 10.4103/ijmpo.ijmpo_113_19

Quick Response Code:

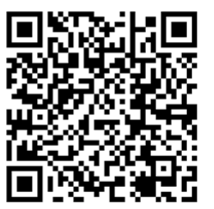




\section{Laboratory data}

Hemoglobin $(\mathrm{Hb})$, total leukocyte count (TLC), platelet count, erythrocyte sedimentation rate (ESR), lactate dehydrogenase (LDH) levels, number of blasts in peripheral blood, and bone marrow studies.

\section{Definitions of quantitative hematological parameters}

Anemia: $\mathrm{Hb}<10$ gm\%; leukopenia: TLC <4 × 109/L; leukocytosis: TLC $>12 \times 109 / \mathrm{L} ; \quad$ neutropenia: absolute neutrophil count (ANC) <1 $\times 109 / \mathrm{L}$; thrombocytopenia: platelets $<100 \times 109 / \mathrm{L}$; thrombocytosis: platelets $>400 \times 109 / \mathrm{L}$; upper limit for serum LDH: $500 \mathrm{IU} / \mathrm{L}$; high ESR: $>20 \mathrm{~mm} 1^{\text {st }} \mathrm{h}$; and acute leukemia: $>20 \%$ blasts in peripheral blood or bone marrow.

\section{Statistical analysis}

Data analysis was done, and the results were compared between the two groups. Using Fisher's exact test with two-sided tests and 5\% level of significance, differences in frequencies were tested for statistical significance.

\section{Results}

The total number of patients in the study group was 63. Clinical and laboratory data of all the cases were analyzed, and the study group was divided into leukemic and nonleukemic groups based on bone marrow diagnosis. Of these, $30.15 \%(n=19)$ of 63 cases were diagnosed with leukemia and the rest of the cases accounting to $69.85 \%(n=44)$ fell into the nonleukemic group who were diagnosed and treated as JIA. Among patients diagnosed with leukemia, ALL was the most common diagnosis seen in $89.48 \%(n=17)$ of cases. Among the nonleukemic group, $59.09 \%(n=26)$ of cases showed normal marrow on bone marrow examination, as depicted in Table 1.

\section{Demographic data}

The median age at diagnosis was significantly higher in the leukemic group compared to the nonleukemic group (9 [4-16] vs. 6.1 [2-15] years, respectively) with $P=0.0118$. No statistical difference was observed in gender for both the groups, with $\mathrm{M}$ : F of 1.5-1:1.

\begin{tabular}{lc}
\hline \multicolumn{1}{c}{$\begin{array}{c}\text { Table 1: Distribution of cases in the study group into } \\
\text { leukemic and nonleukemic groups }\end{array}$} \\
\hline Sub group & No. of cases (\%) \\
\hline Leukemic group & $19(30.15)$ \\
ALL & $17(89.48)$ \\
AML & $2(10.52)$ \\
Nonleukemic group & $44(69.85)$ \\
Normal marrow & $26(59.09)$ \\
Reactive marrow & $18(40.91)$ \\
Total number of cases & $63(100)$ \\
\hline ALL - Acute lymphoblastic leukemia; AML - Acute myeloid \\
leukemia
\end{tabular}

\section{Clinical data}

The occurrence of clinical manifestations in both the groups was assessed and compared, as shown in Table 2. Fever was the most common presenting complaint and was present in all the patients of both the groups, and there were no differences observed in the frequencies of arthralgia in both the groups. The occurrence of hepatomegaly and splenomegaly was comparatively higher in the leukemic group than in patients with JIA $(42.1 \%$ vs. $4.54 \%, P=0.008$, and $47.36 \%$ vs. $6.81 \%, P=0.0006$, respectively). On comparison of musculoskeletal manifestations, the predominant type of arthritis was oligoarticular (68.15\%) in the leukemic group and polyarticular $(77.27 \%)$ in the nonleukemic group. The occurrence of lymphadenopathy was almost equal in both the groups with an insignificant $P$ value $(P=0.9878)$. Rheumatoid rash was noted in $20 \%$ of JIA patients, and none of the patients in the leukemic group had rash.

\section{Laboratory data}

The laboratory parameters between the two study groups were assessed, and the median hemoglobin, white blood cell count, ANC, and platelet count were significantly lower in leukemia patients than in JIA patients $\left(6.7 \mathrm{~g} / \mathrm{dl}\right.$ vs. $10.2 \mathrm{~g} / \mathrm{dl}, 4400 / \mathrm{mm}^{3}$ vs. $15,600 / \mathrm{mm}^{3}, 0.7 \times 103 / \mathrm{mm}^{3}$ vs. $6.9 \times 103 / \mathrm{mm}^{3}$, and $56,000 / \mathrm{mm}^{3}$ vs. 3.8 lakhs $/ \mathrm{mm}^{3}$, respectively).

The percentages of anemia, leukopenia, and thrombocytopenia were statistically higher in leukemia patients than in JIA patients $(36.84 \%$ vs. $9.09 \%, P 0.0313$; $47.3 \%$ vs. $13.63 \%, P 0.0081$; and $57.89 \%$ vs. $9.09 \%$, $P<0.0001$, respectively). Neutropenia was seen in $78.94 \%$ of cases in the leukemic group, and none of the cases in the nonleukemic group had neutropenia. In contrast to the leukemic group, the frequencies of leukocytosis were higher in JIA patients and $40.91 \%$ of cases showed reactive marrow [Figure 1]. All cases with leukemia showed thrombocytopenia, whereas thrombocytosis was seen only in nonleukemic patients, as shown in Table 3. Increased LDH levels were seen in $63 \%$ of leukemia patients, and high ESR was noted in $75 \%$ of JIA patients.

Of 19 cases of leukemia, 17 were diagnosed as ALL and 2 as acute myeloid leukemia. Seven patients (36.8\%) presented with peripheral pancytopenia with no blasts in peripheral blood [Figure 2] and 12 patients (63.2\%) presented with bi/monocytopenia and few blasts in the peripheral blood. Immunophenotyping revealed that the frequencies of the presence of musculoskeletal manifestations were similar in both B- Acute lymphoblastic leukemia and T-Acute lymphoblastic leukemia.

\section{Discussion}

Leukemia is the most common pediatric cancer in many countries such as India, Brazil, Canada, and the United States. ${ }^{[1-3]}$ More than $80 \%$ of all childhood cancer cases 


\begin{tabular}{|c|c|c|c|}
\hline Clinical feature & Leukemia group $(n=19), n(\%)$ & Nonleukemic group/JIA $(n=44), n(\%)$ & $P$ \\
\hline Fever & $19(100)$ & $44(100)$ & - \\
\hline Oligoarticular arthritis & $13(68.15)$ & $10(22.72)$ & $0.0015^{*}$ \\
\hline Polyarticular arthritis & $6(31.5)$ & $34(77.27)$ & $0.0015^{*}$ \\
\hline Hepatomegaly & $8(42.1)$ & $2(4.54)$ & $0.008 *$ \\
\hline Splenomegaly & $9(47.36)$ & $3(6.81)$ & $0.0006^{*}$ \\
\hline Lymphadenopathy & $5(26.31)$ & $10(22.72)$ & 0.9878 \\
\hline Rash & 0 & $9(20)$ & 0.0824 \\
\hline
\end{tabular}

JIA - Juvenile idiopathic arthritis

\begin{tabular}{|c|c|c|c|}
\hline Laboratory parameter & Leukemic group $(n=19), n(\%)$ & Nonleukemic group/JIA $(n=44), n(\%)$ & $\boldsymbol{P}$ \\
\hline Severe anemia & $7(36.84)$ & $4(9.09)$ & $0.0313 *$ \\
\hline Leukopenia & $9(47.3)$ & $6(13.63)$ & $0.0081^{*}$ \\
\hline Neutropenia & $15(78.94)$ & 0 & $<0.0001 *$ \\
\hline Leukocytosis & $3(15.78)$ & $17(38.63)$ & 0.0862 \\
\hline Neutrophilia & 0 & $21(47.72)$ & $<0.0001 *$ \\
\hline Thrombocytopenia & $11(57.89)$ & $4(9.09)$ & $<0.0001 *$ \\
\hline Thrombocytosis & 0 & $6(13.63)$ & 0.1658 \\
\hline High ESR & 0 & $33(75)$ & $<0.0001 *$ \\
\hline High LDH & $12(63.15)$ & 0 & $<0.0001 *$ \\
\hline
\end{tabular}

JIA - Juvenile idiopathic arthritis; ESR - Erythrocyte sedimentation rate; LDH - Lactate dehydrogenase

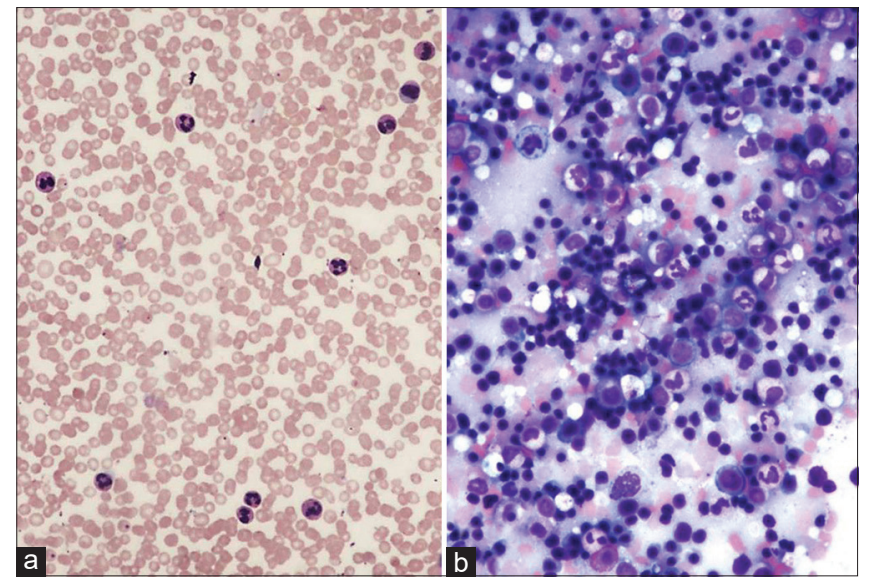

Figure 1: (a) Juvenile idiopathic arthritis - peripheral smear shows neutrophilic leukocytosis - Giemsa, $\times 100$. (b) Reactive marrow and absence of blasts - Giemsa, $\times 100$

occur in low- and middle-income countries. ${ }^{[4]}$ In India, the highest incidence was seen in Delhi at age-adjusted incidence rates of $101.3 /$ million in boys and $62.3 /$ million in girls. ${ }^{[5]}$ The clinical picture of acute leukemia in children constitutes varied manifestations unlike adults. The disease can present with musculoskeletal findings mimicking JIA, rheumatic fever, juvenile systemic lupus erythematosus, and vasculitis. ${ }^{[6-13]}$

The present study was done to evaluate and investigate the significance of musculoskeletal features as a presenting manifestation of leukemia in children who were misdiagnosed as JIA. JIA is the most common chronic rheumatologic disease in children. Characteristic features

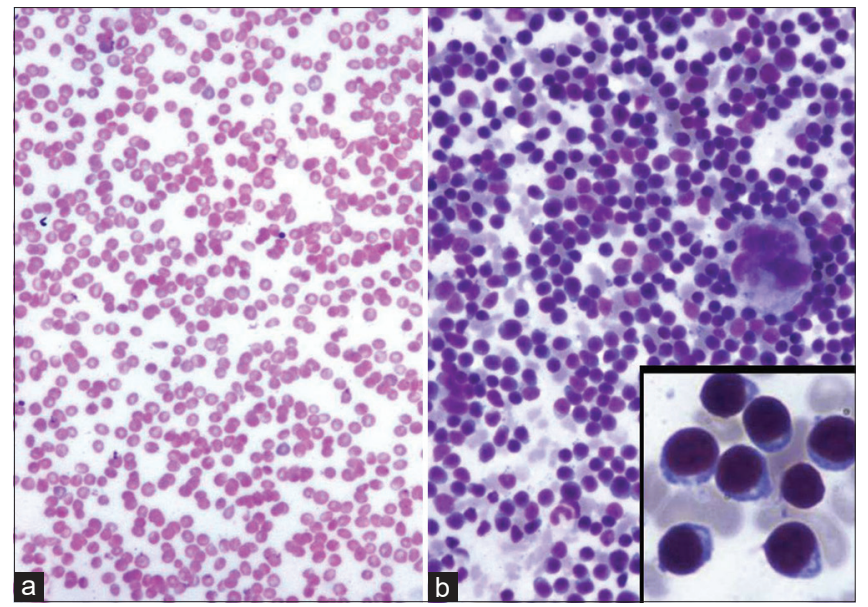

Figure 2: Acute leukemia (a) Peripheral pancytopenia - Giemsa, $\times 100$. (b) Hypercellular marrow with prominence of blasts - Giemsa, $\times 100$ (inset shows blasts - Giemsa, $\times 400$ )

of JIA include arthritis for at least 6 weeks, morning stiffness, joint pain or abnormal joint use, spiking fevers, and evanescent rash on the trunk and extremities. ${ }^{[14,15]}$

Arthritis or arthritis-like symptoms may be seen in other conditions, and leukemia is the single most important differential diagnosis mistaken for JA. Children with leukemia can present with various osteoarticular signals and symptoms, including arthralgia, arthritis, and limb pain, which are typically severe and may awaken the child from sleep. This is due to the expansion of lymphoblasts in bone metaphyses. ${ }^{[15,16]}$

The subtypes of JIA include systemic-onset JIA (SoJIA), oligoarticular JIA, polyarticular JIA, psoriatic arthritis, 
enthesitis-related arthritis, and undifferentiated arthritis. SoJIA is an infrequent subtype of JIA and has prominent extra-articular manifestations and a significant elevation in acute-phase reactants. ${ }^{[17]}$ At disease onset, patients with SoJIA may have only systemic manifestations without arthritis, thereby causing diagnostic delay. The possibility of leukemia should be always considered in the differential diagnosis of SoJIA presenting with following atypical, clinical, and laboratory features.

Atypical features of systemic-onset juvenile idiopathic arthritis favoring leukemia

\section{Clinical features}

Pain disproportionate to severity of arthritis and predominantly nocturnal, nonarticular bony pain requiring opiate analgesia, sequential improvement with steroids initially with subsequent rapid deterioration, and asymmetrical pauciarticular arthritis. ${ }^{[18]}$

\section{Laboratory features}

Severe anemia, leukopenia, neutropenia with relative lymphocytosis, thrombocytopenia, elevated serum LDH, and acute-phase response disproportionate to severity of arthritis.

In our study, the presence of fever, hepatosplenomegaly, limb pain, and weight loss was significantly noted in $31.5 \%$ of patients with leukemia than in JIA patients. These findings significantly correlated with Tamashiro et al. and Brix et al. studies. ${ }^{[18-21]}$

We also found higher levels of anemia, leukopenia, neutropenia, and thrombocytopenia, in patients with leukemia which was similar to the findings in other studies. Thrombocytopenia with neutropenia and lymphocytosis is rare in children with JIA, and their presence suggests the possibility of leukemia. Tamashiro et al. found in their study that limb pain and thrombocytopenia are two independent variables associated with leukemia in $91 \%$ of their patients. ${ }^{[18]} \mathrm{LDH}$ is a marker of increased cell turnover and increased LDH was seen in $63.15 \%$ of cases of leukemia which correlates with a study by Brix et al. and Wallendal et al., who found that serum LDH levels were significantly higher in cancer patients, including leukemia. ${ }^{[22]}$ A history of nighttime pain was seen in $72.27 \%$ of our cases diagnosed with leukemia. The three most important predictive factors for a pediatric leukemia diagnosis as suggested by Jones et al. are leukopenia, thrombocytopenia, and a history of nighttime pain. ${ }^{[23]}$ In the present study, among the nonleukemic group, fever and rheumatoid rash were predominantly observed in JIA patients. Laboratory data revealed that leukocytosis and thrombocytosis were frequently seen in patients with JIA that could distinguish them from leukemia patients. In the leukemic group, $63.2 \%$ of cases showed blasts in periphery. Brix et al. showed that $70 \%$ of the children with ALL with joint involvement had blasts in peripheral blood at admission equivalent to the children with ALL without joint involvement. ${ }^{[19]}$ This discrepancy could be due to the diagnosis of the disease at different stages. A change in methodology over time such as usage of flow cytometry, which is more sensitive to blasts than morphology, also explains the discrepancy.

Our cases were initially diagnosed as JIA based on clinical findings and started on steroids. A study by Barbosa et al. showed that only $13 \%$ had clinical evidence of arthritis, at the onset of leukemia, of which $8 \%$ were misdiagnosed with rheumatic fever or JIA before referral, and some of these patients had already received steroids, delaying the start of appropriate treatment. ${ }^{[6]}$ Treatment with steroids causes a left shift in the marrow, thereby diagnosis of leukemia was delayed by $1-8$ months. Majority (76.47\%) of the leukemic group were not showing blasts in the peripheral blood and were diagnosed only after bone marrow studies. The importance of investigating leukemia in children and adolescents who present with musculoskeletal manifestations, especially arthritis and thrombocytopenia, is emphasized in this study.

\section{Conclusion}

Bone marrow studies are a prerequisite in diagnosing leukemia in cases with musculoskeletal symptoms and absence of blasts in peripheral blood. Leukemia must be excluded first, as treatment with steroids temporarily reduces the cell counts and thereby masks the symptoms. This further delays a malignancy diagnosis and reduces the subsequent response to chemotherapy. In this study, a few atypical, clinical, and laboratory parameters were demonstrated that differentiate acute leukemia from JIA at disease onset, thereby reducing diagnostic delay.

\section{Financial support and sponsorship}

Nil.

\section{Conflicts of interest}

There are no conflicts of interest.

\section{References}

1. Linabery AM, Ross JA. Trends in childhood cancer incidence in the U.S. (1992-2004). Cancer 2008;112:416-32.

2. de Camargo B, de Oliveira Santos M, Rebelo MS, de Souza Reis R, Ferman S, Noronha CP, et al. Cancer incidence among children and adolescents in Brazil: First report of 14 population-based cancer registries. Int J Cancer 2010;126:715-20.

3. Helmick CG, Felson DT, Lawrence RC, Gabriel S, Hirsch R, Kwoh CK, et al. Estimates of the prevalence of arthritis and other rheumatic conditions in the United States. Part I. Arthritis Rheum 2008;58:15-25.

4. Barr R, Riberio R, Agarwal B, Masera G, Hesseling P, Magrath I. Pediatric oncology in countries with limited resources. In: Pizzo PA, Poplack DG, editors. Principles and Practice of Pediatric Oncology. $5^{\text {th }}$ ed. Philadelphia: Lippincott Williams and Wilkins; 2006. p. 1605-17.

5. Bashar A. Incidence and pattern of childhood cancers in India: 
Findings from population-based cancer registries. Indian $\mathrm{J}$ Cancer 2016;53:511-2.

6. Barbosa CM, Nakamura C, Terreri MT, Lee ML, Petrilli AS, Hilário MO. Musculoskeletal manifestations as the onset of acute leukemias in childhood. J Pediatr (Rio J) 2002;78:481-4.

7. Robazzi TC, Barreto JH, Silva LR, Santiago MB, Mendonça N. Osteoarticular manifestations as initial presentation of acute leukemias in children and adolescents in Bahia, Brazil. J Pediatr Hematol Oncol 2007;29:622-6.

8. Campos LM, Goldstein S, Santiago RA, Jesus AA, Cristofani LM, Odone Filho $\mathrm{V}$, et al. Musculoskeletal involvement as a first manifestation of neoplasm disease. Rev Assoc Med Bras (1992) 2008;54:132-8.

9. Schaller J. Arthritis as a presenting manifestation of malignancy in children. J Pediatr 1972;81:793-7.

10. Saulsbury FT, Sabio H. Acute leukemia presenting as arthritis in children. Clin Pediatr (Phila) 1985;24:625-8.

11. Goncalves M, Terreri MT, Barbosa CM, Len CA, Lee L, Hilario MO. Diagnosis of malignancies in children with musculoskeletal complaints. Sao Paulo Med J 2005;123:21-3.

12. Murray MJ, Tang T, Ryder C, Mabin D, Nicholson JC. Childhood leukaemia masquerading as juvenile idiopathic arthritis. BMJ 2004;329:959-61.

13. Tafaghodi F, Aghighi Y, Rokni Yazdi H, Shakiba M, Adibi A. Predictive plain X-ray findings in distinguishing early stage acute lymphoblastic leukemia from juvenile idiopathic arthritis. Clin Rheumatol 2009;28:1253-8.

14. Marwaha RK, Kulkarni KP, Bansal D, Trehan A. Acute lymphoblastic leukemia masquerading as juvenile rheumatoid arthritis: Diagnostic pitfall and association with survival. Ann Hematol 2010;89:249-54.

15. Lovell DJ. Juvenile idiopathic arthritis: Clinical features. In:
Kippel JH, Stone JH, Crofford LJ, White PH, editors. Primer on the Rheumatic Diseases. $13^{\text {th }}$ ed. New York: Springer Science; 2008.

16. Simard JF, Neovius M, Hagelberg S, Askling J. Juvenile idiopathic arthritis and risk of cancer: A nationwide cohort study. Arthritis Rheum 2010;62:3776-82.

17. Yanagimachi M, Miyamae $T$, Naruto $T$, Hara $T$, Kikuchi $M$, Hara R, et al. Association of HLA-A*02:06 and HLA-DRB $1 * 04: 05$ with clinical subtypes of juvenile idiopathic arthritis. J Hum Genet 2011;56:196-9.

18. Tamashiro MS, Aikawa NE, Campos LM, Cristofani LM, Odone-Filho V, Silva CA. Discrimination of acute lymphoblastic leukemia from systemic-onset juvenile idiopathic arthritis at disease onset. Clinics (Sao Paulo) 2011;66:1665-9.

19. Brix N, Rosthøj S, Herlin T, Hasle H. Arthritis as presenting manifestation of acute lymphoblastic leukaemia in children. Arch Dis Child 2015;100:821-5.

20. Mulder H, Herregods N, Mondelaers V, Benoit Y, De Moerloose B. Musculoskeletal manifestations in children with acute lymphoblastic leukaemia. Belg J Hematol 2012;3:3-11

21. Jali S, Prashanth GP, Amarkhed P. Migratory polyarthritis in aleukemic lymphoblastic leukemia: An undesignated paraneoplastic syndrome. J Sci Soc 2013;40:44-6.

22. Wallendal M, Stork L, Hollister JR. The discriminating value of serum lactate dehydrogenase levels in children with malignant neoplasms presenting as joint pain. Arch Pediatr Adolesc Med 1996;150:70-3.

23. Jones OY, Spencer CH, Bowyer SL, Dent PB, Gottlieb BS, Rabinovich CE. A multicenter case-control study on predictive factors distinguishing childhood leukemia from juvenile rheumatoid arthritis. Pediatrics 2006;117:e840-4. 